\title{
Erratum: Hard-Hexagon Model: Anisotropy of Correlation Length and Interfacial Tension ${ }^{1}$
}

\section{Masafumi Fujimoto ${ }^{2}$}

\author{
Received September 19, 1990
}

The following corrections should be made in the above paper.

1. In the rhs of (2.7) two tildes were missing:

$$
\left\langle\sigma_{00} \sigma_{l m}\right\rangle-\left\langle\sigma_{00}\right\rangle\left\langle\sigma_{l m}\right\rangle=\sum_{p \neq 1} \widetilde{S}_{0}(1, p) \widetilde{S}_{m}(p, 1)\left[\frac{V(p)}{V(1)}\right]^{l}
$$

2. A right square bracket in the third line of $(2.34 a)$ should be moved to the fourth line:

$$
\begin{aligned}
\left\langle\sigma_{00} \sigma_{l m}\right\rangle & -\left\langle\sigma_{00}\right\rangle\left\langle\sigma_{l m}\right\rangle \\
\sim & \alpha \exp \left(-\frac{r}{\xi}\right) \cos \left\{\frac{2 \pi}{3}(l+m)\right. \\
+ & {\left[-4 \sqrt{3} \cos \theta_{||} \cos \left(\theta_{||}+\frac{\pi}{3}\right) \cos \left(\theta_{||}-\frac{\pi}{3}\right) \exp \left(-\frac{10}{6} \varepsilon\right)\right.} \\
+ & \left.\left.O\left[\exp \left(-\frac{20}{6} \varepsilon\right)\right]\right]+\delta\right\}
\end{aligned}
$$

3. The equation number $(2.32 \mathrm{~b})$ in line 5 from the bottom of p. 1364 should be replaced by $(2.34 b)$.

4. A boldface $\mathbf{R}$ in the lhs of (3.31a) should be replaced by $R$ :

$$
\lambda R(\mathbf{r})=\min _{\mathbf{n}} \frac{\sigma(\mathbf{n})}{(\mathbf{r} \cdot \mathbf{n})}
$$

\footnotetext{
${ }^{1}$ This paper appeared in J. Stat. Phys. 59:1355 (1990).

${ }^{2}$ Faculty of Science, Osaka University, Toyonaka, Osaka, 560 Japan.
} 
5. In the lhs of the inequality in (3.34a) $\theta_{\perp}$ should be removed:

$$
a_{s}\left(\theta_{\perp}\right) \sim \frac{\sin \left(\theta_{\perp}-\pi / 3\right)}{\sin \left(\theta_{\perp}+\pi / 3\right)} \quad \text { for } \quad-\frac{\pi}{3}<\theta_{\perp}<\frac{\pi}{3}
$$

6. In the rhs of (3.42) a factor 2 was missing:

$$
\frac{\rho}{R}=\frac{[2 /(3 \sqrt{3})]\left[f^{4}\left(x, x^{3}\right) / f^{4}\left(-x, x^{3}\right)\right]}{-(2 / \sqrt{3}) \ln \left[x^{1 / 3} f\left(-1, x^{3}\right) / f\left(-x, x^{3}\right)\right]}
$$

